\title{
Impedance Controller Design of Internet-Based Teleoperation Using Absolute Stability Concept
}

\author{
Hyun Chul Cho and Jong Hyeon Park \\ School of Mechanical Engineering, Hanyang University \\ Seoul, 133-791, Korea \\ email: hcho@ihanyang.ac.kr,jongpark@hanyang.ac.kr
}

\begin{abstract}
Relationships between impedance parameters and scaling factors are derived using absolute stability and passivity. For this, the teleoperation system is represented as a two-port model with a hybrid matrix. Design procedure of the impedance parameters for the absolute stability of the teleoperator under the time delay is proposed. The validity of the proposed control scheme is demonstrated by experiments with a 1-dof master/slave system connected through the Internet.
\end{abstract}

\section{Introduction}

Impedance control adjusts the impedance of the manipulator, which is defined as $Z(s)=F(s) / V(s)$ where $F(s)$ denotes force and $V(s)$ denotes velocities in the Laplace transform; and is determined typically by an inertia, a damper, and a spring. The desired impedance of the manipulator depends on the task that the manipulator performs.

In many telerobotic tasks, robot manipulators interact with their environments. Excessive contact force between the robot and the environment should be prevented for the stability of the controlled system and avoiding system damages. Besides, the tracking ability in a freespace cannot be neglected for the task performance. Since the impedance control can treat these situations effectively, it has many applications for a teleoperation $[1,2,3]$.

Two-port model, found in the electrical network theory, has been used for describing stability and performance in teleoperation $[7,8,9,10]$. Using the mechanical analog to the electrical two-port, this model characterizes the energy exchanges between the human operator and the environment, and this property is useful in investigating the stability of the entire teleoperation system.

This paper presents relations among design parameters for stability and design procedures of the impedance parameters. Stability analysis is performed with absolute stability and passivity, assuming the human operator and environment are passive. Analysis results show that absolute stability is a less conservative method for the stability analysis than passivity. A design procedure of the impedance controllers are summarized to satisfy of the teleoperation system under time delay. Several experiments with the designed impedance parameters shows good performance.

Section 2 summarizes the impedance controllers and some definitions. Section 3 describes the stability analysis, and parameter design procedure is shown in Section 4. Experiments with a 1-dof teleoperation system and their results are shown in Section 5, followed by conclusions in Section 6 .

\section{Controller Design}

In this section, impedance controllers for the master and the slave are derived.

\subsection{Dynamics of Master and Slave}

The dynamics of the single dof master/slave system are modeled as a mass-damper system as follows:

$$
\begin{aligned}
m_{m} \ddot{x}_{m}(t)+b_{m} \dot{x}_{m}(t) & =u_{m}(t)+f_{h}(t) \\
m_{s} \ddot{x}_{s}(t)+b_{s} \dot{x}_{s}(t) & =u_{s}(t)-f_{e}(t)
\end{aligned}
$$

where $x$ and $u$ denote position and torque; $m$ and $b$ denote mass and viscous coefficient; subscript ' $m$ ' and ' $s$ ' denote the master and the slave, respectively; $f_{h}$ is the force applied at the master by the operator, and $f_{e}$ is the force exerted on the slave by its environment.

\subsection{Delayed Signals and Scaling Factors}

When there is a time delay in the communication channel, the signals from and to the channel are re- 
lated as:

$$
\begin{array}{rlll}
x_{m}^{d}(t) & :=x_{m}\left(t-T_{1}\right), & \dot{x}_{m}^{d}(t) & :=\dot{x}_{m}\left(t-T_{1}\right) \\
f_{h}^{d}(t) & :=f_{h}\left(t-T_{1}\right), & f_{e}^{d}(t) & :=f_{e}\left(t-T_{2}\right)
\end{array}
$$

where $x_{m}^{d}, \dot{x}_{m}^{d}$, and $f_{h}^{d}$ are the position, velocity commands and the operating force, respectively, transmitted from the master to the slave through the communication; $f_{e}^{d}$ is the external force at the slave transmitted from the slave to the master; $T_{1}$ is the time delay of the signal flowing from the master to the slave, and $T_{2}$ is in the opposite direction.

These delayed signal out of the communication block is then scaled down or up with some factors depending on the application. Using the scaling factors, the position/velocity command to the slave and the force command to the master are modified such that

$$
x_{s}=k_{p} x_{m}^{d}, \quad f_{h}=k_{f} f_{e}^{d}
$$

where $k_{p}$ and $k_{f}$ are the position and force scaling factors, respectively.

\subsection{Impedance Controllers for Master and Slave}

In order to derive impedance control input for the master, suppose that the desired impedance for the master is specified by

$$
\bar{m}_{m} \ddot{x}_{m}(t)+\bar{b}_{m} \dot{x}_{m}(t)+\bar{k}_{m} x_{m}(t)=f_{h}(t)-k_{f} f_{e}^{d}(t)
$$

where $\bar{m}_{m}, \bar{b}_{m}$ and $\bar{k}_{m}$ are the desired inertia, damping coefficient, and stiffness, respectively.

Combining Eqs. (1) and (3) to remove acceleration $\ddot{x}_{m}$ results in the control input to the master:

$$
\begin{aligned}
u_{m}(t)= & \left(b_{m}-\frac{m_{m}}{\bar{m}_{m}} \bar{b}_{m}\right) \dot{x}_{m}(t)+\left(\frac{m_{m}}{\bar{m}_{m}}-1\right) f_{h}(t) \\
& -\frac{m_{m}}{\bar{m}_{m}}\left\{k_{f} f_{e}^{d}(t)+\bar{k}_{m} x_{m}(t)\right\} .
\end{aligned}
$$

The desired impedance for the slave is also defined in the similar form.

$$
\bar{m}_{s} \ddot{\tilde{x}}_{s}(t)+\bar{b}_{s} \dot{\tilde{x}}_{s}(t)+\bar{k}_{s} \tilde{x}_{s}(t)=-f_{e}(t)
$$

where $\bar{m}_{s}, \bar{b}_{s}$ and $\bar{k}_{s}$ are the desired inertia, damping coefficient, and stiffness, respectively; $\tilde{x}_{s}:=x_{s}-$ $k_{p} x_{m}^{d}$. From Eqs. (2) and (4), the control input for

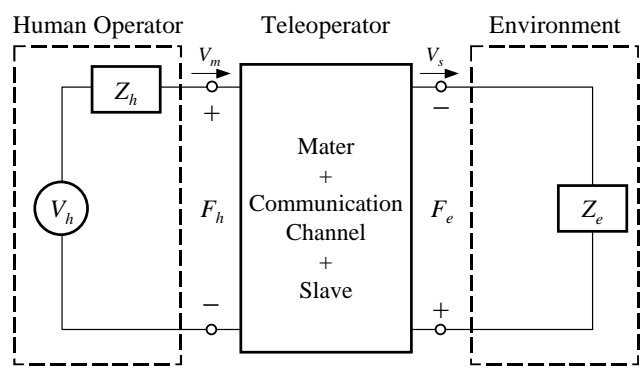

Figure 1: Two-port model of the system.

the slave can be found:

$$
\begin{aligned}
u_{s}(t)= & \left(b_{s}-\frac{m_{s}}{\bar{m}_{s}} \bar{b}_{s}\right) \dot{x}_{s}(t)-\frac{m_{s}}{\bar{m}_{s}} \bar{k}_{s} \cdot x_{s}(t) \\
& +m_{s} k_{p}\left(\frac{\bar{b}_{s}}{\bar{m}_{s}}-\frac{\bar{b}_{m}}{\bar{m}_{m}}\right) \dot{x}_{m}^{d}(t) \\
& +m_{s} k_{p}\left(\frac{\bar{k}_{s}}{\bar{m}_{s}}-\frac{\bar{k}_{m}}{\bar{m}_{m}}\right) x_{m}^{d}(t)+\frac{m_{s} k_{p}}{\bar{m}_{m}} f_{h}^{d}(t) \\
& +\frac{\bar{m}_{m}-m_{s}}{\bar{m}_{m}} f_{e}(t)-\frac{m_{s}}{\bar{m}_{m}} k_{p} k_{f} \cdot f_{e}^{d d}(t)
\end{aligned}
$$

where $f_{e}^{d d}(t):=f_{e}\left(t-T_{1}-T_{2}\left(t-T_{1}\right)\right)$.

\section{Absolute Stability and Passivity}

Absolute stability and passivity concept provide a simple tool to analyze the stability of a system based only on input-output properties of the system. Thus, these methods are suitable for the stability analysis of a two-port teleoperation having unmodeled passive human operators and environments.

\subsection{Definitions}

Two-Port Model Representation. In Fig. 1, the teleoperation system is modeled as a two-port network, where the operator-master interface is designated as the master port and the slave-environment interface as the slave port.

The relationship between efforts $\left(f_{h}, f_{e}\right)$ and flows $\left(\dot{x}_{m}, \dot{x}_{s}\right)$ of the two ports can be described in terms of the so-called hybrid matrix obtained from the properly controlled master and slave behaviors of Eqs. (3) and (4). The hybrid matrix for the teleoperation system and its parameters are as follows.

$$
\left[\begin{array}{l}
F_{h}(s) \\
V_{s}(s)
\end{array}\right]=\left[\begin{array}{ll}
h_{11} & h_{12} \\
h_{21} & h_{22}
\end{array}\right]\left[\begin{array}{c}
V_{m}(s) \\
-F_{e}(s)
\end{array}\right]
$$

where $F_{h}(s), V_{m}(s), V_{s}(s)$ and $F_{e}(s)$ are the Laplace transforms of $f_{h}(t), \dot{x}_{m}(t), \dot{x}_{s}(t)$, and $f_{e}(t)$, respectively, and $h$-parameters are 


$$
\begin{gathered}
h_{11}=\bar{m}_{m} s+\bar{b}_{m}+\frac{\bar{k}_{m}}{s}, \quad h_{12}=-k_{f} \cdot e^{-T_{2} s}, \\
h_{21}=k_{p} \cdot e^{-T_{1} s}, \quad h_{22}=\frac{s}{\bar{m}_{s} s^{2}+\bar{b}_{s} s+\bar{k}_{s}} .
\end{gathered}
$$

Absolute Stability. Absolute stability [4] is defined as follows.

Definition: A linear two-port is said to be absolutely stable if there exists no set of passive terminating one-port impedances for which the system is unstable. If the network is not absolutely stable, it is potentially unstable.

A necessary and sufficient condition for the absolute stability of a two-port network is that one-port networks, resulting from any passive output and input termination, are themselves passive [4]. Llewellyn's stability criteria [5] provides necessary and sufficient conditions for the absolute stability, and from this criteria, a two-port network is absolutely stable if and only if

a) $h_{11}$ and $h_{22}$ have no poles in the right half plane;

b) Any poles of $h_{11}$ and $h_{22}$ on the imaginary axis are simple with real and positive residues;

c) For all real values of $\omega$,

$$
\begin{gathered}
\operatorname{Re} h_{11} \geq 0, \quad \operatorname{Re} h_{22} \geq 0, \\
2 \operatorname{Re} h_{11} \operatorname{Re} h_{22}-\operatorname{Re}\left(h_{12} h_{21}\right)-\left|h_{12} h_{21}\right| \geq 0 .
\end{gathered}
$$

Passivity. The passivity formalism represents a mathematical description of the intuitive physical concepts of power and energy. Intuitively, a system is passive if it absorbs more energy than it produces, and the passivity can be defined formally as follows.

Definition: A two-port is said to be passive if for all excitations the total energy delivered to the network, $E(t)=\int_{-\infty}^{t}\left[f_{h}(\tau) \dot{x}_{m}(\tau)-f_{e}(\tau) \dot{x}_{s}(\tau)\right] d \tau$, at its input and output ports is nonnegative. If the network is not passive, it is active.

In this paper, Raisbeck's passivity criterion [6] is used to check the passivity of the two-port. From Raisbeck's passivity criterion, a linear two-port network is passive if and only if

$\left.\mathrm{a}^{\prime}\right)$ The $h$-parameters have no poles in the RHP;

$\left.\mathrm{b}^{\prime}\right)$ Any poles of the $h$-parameters on the imaginary axis are simple, and the residues of the $h$-parameters at these poles satisfy the following conditions:

$$
k_{11} \geq 0, \quad k_{22} \geq 0, \quad k_{11} k_{22}-k_{12} k_{21} \geq 0
$$

where $k_{i j}$ denotes the residue of $h_{i j}$ at the pole. $\left.c^{\prime}\right)$ For all real values of $\omega$,

$$
\begin{gathered}
\operatorname{Re} h_{11} \geq 0, \quad \operatorname{Re} h_{22} \geq 0, \\
4 \operatorname{Re} h_{11} \operatorname{Re} h_{22}-\left[\operatorname{Re} h_{12}+\operatorname{Re} h_{21}\right]^{2} \\
-\left[\operatorname{Im} h_{12}-\operatorname{Im} h_{21}\right]^{2} \geq 0 .
\end{gathered}
$$

\subsection{Stability Analysis}

The stability of the two-port model is analyzed by the absolute stability and the passivity. The relationships between impedance parameters and scaling factors are derived through this analysis.

Stability Analysis Using Absolute Stability. For the given two-port network, Eq. (5), conditions (a) and (b), together with the first and second conditions in (c) are satisfied with positive master and slave impedance parameters. The last of condition (c) can be expressed by

$\left[\cos \left(T_{1}+T_{2}\right) \omega-1\right] k_{p} k_{f}+\frac{2 \bar{b}_{m} \bar{b}_{s} \omega^{2}}{\left(\bar{k}_{s}-\bar{m}_{s} \omega^{2}\right)^{2}+\left(\bar{b}_{s} \omega\right)^{2}} \geq 0$

which is satisfied only if

$$
k_{p} k_{f} \leq \frac{\bar{b}_{m} \bar{b}_{s} \omega^{2}}{\left(\bar{k}_{s}-\bar{m}_{s} \omega^{2}\right)^{2}+\left(\bar{b}_{s} \omega\right)^{2}}, \quad \forall \omega \geq 0 .
$$

If the designed parameters satisfy Eq. (7), the teleoperation system will be absolutely stable for any set of passive human operators and environments. When there is no time delay, Eq. (6) is always satisfied independently of positive impedance parameters and scaling factors.

Stability Analysis Using Passivity. In a similar way, the passivity of the two-port is investigated. All conditions except the last of $\left(\mathrm{c}^{\prime}\right)$ are satisfied with the positive impedance parameters, and the last condition is represented by

$k_{p}^{2}+k_{f}^{2}-2 k_{p} k_{f} \cos \left(T_{1}+T_{2}\right) \omega \leq \frac{4 \bar{b}_{m} \bar{b}_{s} \omega^{2}}{\left(\bar{k}_{s}-\bar{m}_{s} \omega^{2}\right)^{2}+\left(\bar{b}_{s} \omega\right)^{2}}$

which is satisfied only if

$$
\left(k_{p}+k_{f}\right)^{2} \leq \frac{4 \bar{b}_{m} \bar{b}_{s} \omega^{2}}{\left(\bar{k}_{s}-\bar{m}_{s} \omega^{2}\right)^{2}+\left(\bar{b}_{s} \omega\right)^{2}}, \quad \forall \omega \geq 0 .
$$

The entire teleoperation system including a passive human operator and a passive environment is passive with the parameters satisfying Eq. (9). And for the case of $T_{1}+T_{2}=0$, Eq. (8) becomes: 
Table 1: Relations between impedance parameters and scaling factors for the absolute stability or the passivity, where RTT stands for the round-trip time.

\begin{tabular}{|c|c|c|}
\hline Case & RTT & Condition for Absolute Stability \\
\hline 1 & 0 & always satisfied \\
\hline 2 & $>0$ & $k_{p} \cdot k_{f} \leq \frac{\bar{b}_{m} \bar{b}_{s} \omega^{2}}{\left(\bar{k}_{s}-\bar{m}_{s} \omega^{2}\right)^{2}+\left(\bar{b}_{s} \omega\right)^{2}}$ \\
\hline \hline Case & RTT & Condition for Passivity \\
\hline 3 & 0 & $\left(k_{p}-k_{f}\right)^{2} \leq \frac{4 b_{m} b_{s} \omega^{2}}{\left(\bar{k}_{s}-\bar{m}_{s} \omega^{2}\right)^{2}+\left(\bar{b}_{s} \omega\right)^{2}}$ \\
\hline 4 & $>0$ & $\left(k_{p}+k_{f}\right)^{2} \leq \frac{4 b_{m} b_{s} \omega^{2}}{\left(\bar{k}_{s}-\bar{m}_{s} \omega^{2}\right)^{2}+\left(\bar{b}_{s} \omega\right)^{2}}$ \\
\hline
\end{tabular}

$$
\left(k_{p}-k_{f}\right)^{2} \leq \frac{4 \bar{b}_{m} \bar{b}_{s} \omega^{2}}{\left(\bar{k}_{s}-\bar{m}_{s} \omega^{2}\right)^{2}+\left(\bar{b}_{s} \omega\right)^{2}}, \quad \forall \omega \geq 0 .
$$

\section{Comparison of Absolute Stability and Passiv-}

ity. Conditions for the stability are summarized in Table 1 according to the time delay and the stability analysis method. These conditions are functions of scaling factors, slave impedance parameters and only $\bar{b}_{m}$ in the master impedance parameters.

If there is no time delay, the impedance controlledmaster and slave is always absolutely stable, but always passive only when $k_{p}$ is identical to $k_{f}$. Teleoperation tasks, however, mostly require different scaling factors from each other, and this makes the system to be passive only in the limited frequency ranges depending on designed impedance parameters.

Figure 2 is the regions that scaling factors should satisfy for the stability of each case, where boundaries are drawn with a specific frequency and impedance parameters. In this figure, we can observe that the regions for the absolute stability case (1 and 2) include that of the passivity (3 and 4) in the same RTT condition. This result agrees with the so-called stability-activity diagram [11], and shows that a passive network will always be absolutely stable; absolute stability do not, however, imply that the system is passive.

\section{Impedance Parameter Design}

Since the criteria for absolute stability and passivity depend solely on the $h$-parameters, we can use these criteria for impedance parameter design: first, scaling factors are determined properly according to an application, then impedance parameters are selected to satisfy the relations for the stability. In this section, only case 2 in Table 1 is considered.

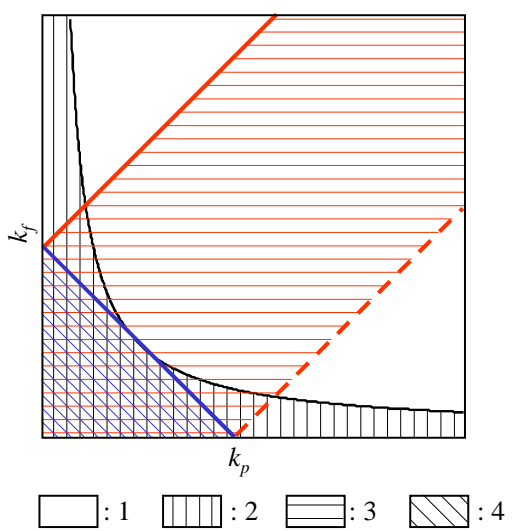

Figure 2: Regions of scaling factors satisfying the stability condition for each case.

Table 2: Coefficients, frequency boundaries, and constraint of $f(\omega)=A \omega^{4}+B \omega^{2}+C \leq 0$ when all slave impedance parameters are positive.

\begin{tabular}{|c||c|}
\hline$A$ & $\bar{m}_{s}^{2}$ \\
\hline$B$ & $\bar{b}_{s}^{2}-\frac{b_{m} b_{s}}{k_{p} k_{f}}-2 \bar{m}_{s} \bar{k}_{s}$ \\
\hline$C$ & $k_{s}^{2}$ \\
\hline Constraints & $B<0, B^{2}>4 A C$ \\
\hline
\end{tabular}

As mentioned earlier, the impedance and scaling parameters should satisfy Eq. (7) for the absolute stability of the teleoperation system under the time delay.

Rearranging Eq. (7) gives a following inequality equation.

$$
f(\omega)=A \omega^{4}+B \omega^{2}+C \leq 0
$$

where $A, B$, and $C$ are summarized in Table 2 . Since $A$ and $C$ are always nonnegative, the coefficient $B$ and the minimum value of $f(\omega), C-\frac{B^{2}}{4 A}$, must be negative in order that the negative $f(\omega)$ could exist even for some frequencies. To satisfy the first condition of $B<0, \bar{b}_{s}$ must be

$$
b_{1}<\bar{b}_{s}<b_{2},
$$

where

$$
b_{1,2}=\frac{\bar{b}_{m} \mp \sqrt{\bar{b}_{m}^{2}+8 k_{p}^{2} k_{f}^{2} \bar{m}_{s} \bar{k}_{s}}}{2 k_{p} k_{f}}
$$

and from the other constraint of $B^{2}>4 A C$, the range of $\bar{b}_{s}$ is

$$
\bar{b}_{s}<b_{3}, \quad b_{4}<\bar{b}_{s}<b_{5}, \quad \bar{b}_{s}>b_{6},
$$


where

$$
\begin{gathered}
b_{3}=\frac{\bar{b}_{m}-\sqrt{\bar{b}_{m}^{2}+16 k_{p}^{2} k_{f}^{2} \bar{m}_{s} \bar{k}_{s}}}{2 k_{p} k_{f}}, \quad b_{4}=0, \\
b_{5}=\frac{\bar{b}_{m}}{k_{p} k_{f}}, \quad b_{6}=\frac{\bar{b}_{m}+\sqrt{\bar{b}_{m}^{2}+16 k_{p}^{2} k_{f}^{2} \bar{m}_{s} \bar{k}_{s}}}{2 k_{p} k_{f}} .
\end{gathered}
$$

From Eqs. (10) and (11) with $\bar{b}_{s}>0$, we can find the range of $\bar{b}_{s}$ satisfying the constraints:

$$
0<\bar{b}_{s}<\frac{\bar{b}_{m}}{k_{p} k_{f}}
$$

Design procedure of the impedance parameters for the absolute stability of the system is summarized below.

1) Select $k_{p}$ and $k_{f}$ properly for the system.

2) Design the master impedance parameters, $\bar{m}_{m}$ and $\bar{k}_{m}>0$, freely with suitable positive $\bar{b}_{m}$.

3) Select $\bar{k}_{s}$ to satisfy a desired compliance of the slave.

4) Select $\bar{b}_{s}$ and $\bar{m}_{s}$ using desired $\zeta, \omega_{n}$ and the inequality of Eq. (12).

\section{Experiments}

In this section, the performance of the designed controller is investigated through the experiments with a 1-dof master/slave bilateral teleoperation system connected to the Internet. At the master side, its human operator pulls and pushes a knob attached to its motor and the force exerted at the master is measured by a load-cell. At the other side, the slave can move straight back and forth with a ball-screw mechanism and a load-cell is installed at its tip, which measures the contact force exerted by the wall.

In order to investigate the performance of the impedance controller under a specific time delay, the communication unit that introduces bilateral time delays is simulated with a memory buffer, in addition to the physical communication line. Data to be exchanged between the master and the slave is written to the buffer. It is sent to its intended destination only at the moment when the buffer is full. Thus, the time delay at the buffer depends on the size of the buffer. In the experiments, the buffer size is fixed at a constant value. Another source of the time delay is the physical communication line. Thus, the average of the time delay depends only on the size of the buffer, and its deviation is exactly that of the actual communication line.

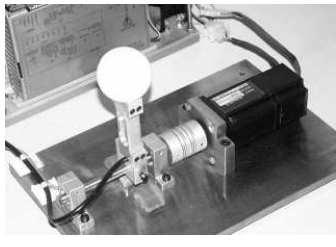

(a) Master

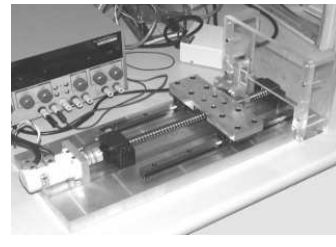

(b) Slave
Figure 3: The master and slave system

Table 3: The parameters used in the experiments

\begin{tabular}{|c|r|c|r|}
\hline$k_{p}$ & $0.1[\mathrm{~m} / \mathrm{rad}]$ & $k_{f}$ & $0.05[\mathrm{~m}]$ \\
\hline \hline $\bar{m}_{m}$ & $0.02\left[\mathrm{kgm}^{2}\right]$ & $\bar{m}_{s}$ & $0.05[\mathrm{~kg}]$ \\
$\bar{b}_{m}$ & $0.05[\mathrm{Nms}]$ & $\bar{b}_{s}$ & $2.1[\mathrm{Ns} / \mathrm{m}]$ \\
$\bar{k}_{m}$ & $0.04[\mathrm{Nm}]$ & $\bar{k}_{s}$ & $80[\mathrm{~N} / \mathrm{m}]$ \\
\hline
\end{tabular}

The impedance parameters used in the experiments are summarized in Table 3. First, the position and force scaling factors are determined to be proper for the system size and configuration. Then, the master impedance parameters, $\bar{m}_{m}, \bar{b}_{m}$, and $\bar{k}_{m}$ are designed considering the maneuverability of the master. The stiffness of the slave impedance model, $\bar{k}_{s}$ is selected according to the desired compliance of the slave, and the mass and damping, $\bar{m}_{s}$ and $\bar{b}_{s}$ are designed with a damping ratio of $\zeta=0.7$ and a natural frequency of $\omega_{n}=30[\mathrm{rad} / \mathrm{s}]$. In this case, $\bar{b}_{s}=2.1<\frac{\bar{b}_{m}}{k_{p} k_{f}}=10$, which satisfies the inequality of Eq. (12).

Figures 4 and 5 show the experimental results when RTT (Round-Trip Time) is about 1.0 or 4.0 second and $\bar{m}_{s}=0.05, \bar{b}_{s}=2.1, \bar{k}_{s}=80$. In spite of a large time delay, the slave shows proper tracking performance, and stable contact behaviors.

Next experiment is performed with the impedance parameters of $\bar{m}_{s}=0.05, \bar{b}_{s}=20, \bar{k}_{s}=80$, which corresponds to $\zeta=5.0, \omega_{n}=40[\mathrm{rad} / \mathrm{s}]$. It is expected to show more stable behavior with the increased damping ratio, but the slave makes the unstable contact though there is relatively small time delay in the communication line (Fig. 6).

\section{Conclusions}

In this paper, relations between impedance parameters and scaling factors are derived using the absolute stability and the passivity concept. If there is no time delay, the impedance-controlled master and slave is always absolutely stable, but it is always passive only when $k_{p}$ is identical to $k_{f}$.

If there is time delay in the communication line, some impedance parameter sets may make the teleopera- 

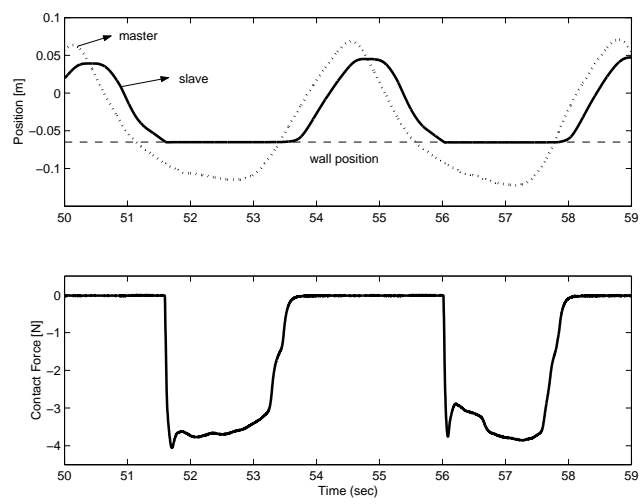

Figure 4: Scaled master/slave positions and external force when $\bar{m}_{s}=0.05, \bar{b}_{s}=2.1, \bar{k}_{s}=80$ and $R T T \approx 1.0 \mathrm{~s}$.
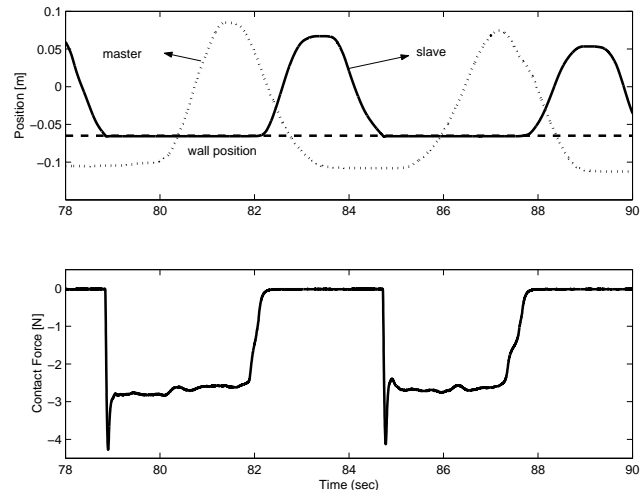

Figure 5: Scaled master/slave position and external force when $\bar{m}_{s}=0.05, \bar{b}_{s}=2.1, \bar{k}_{s}=80$ and $R T T \approx 4.0 \mathrm{~s}$.

tion system unstable regardless of large damping ratio, $\zeta$ in the desired impedance model. And this fact can be observed in the experiment.

Although absolute stability is a less conservative tool to treat the stability of a two-port than the passivity concept, it remains a conservative method due to its allowance for the infinite human impedance. In a future work, less conservative stability analysis will be performed by limiting a maximum level of the human impedance.

\section{References}

[1] R. V. Dubey, T. F. Chan, and S. E. Everett, "Variable Damping Impedance Control of a Bilateral Telerobotic System," IEEE Control Systems, pp. 37-44, 1997.

[2] A. Rubio, A. Avello and J. Florez, "Adaptive Impedance Modification of a Master-Slave Manipulator," IEEE Int. Conf. on Robotics and Automation, pp. 1794-1796, 1999.
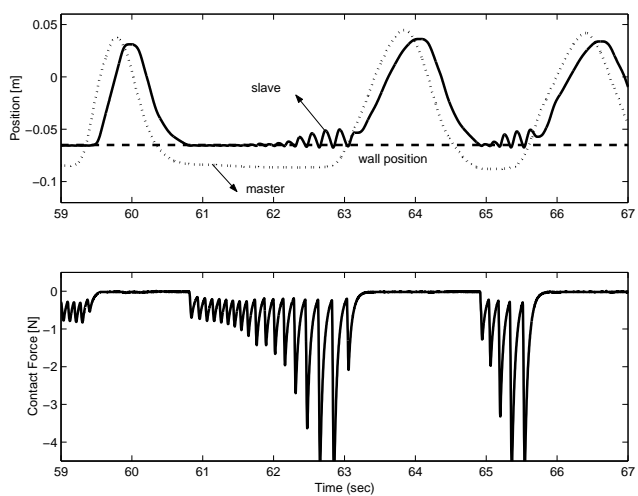

Figure 6: Scaled master/slave position and external force when $\bar{m}_{s}=0.05, \bar{b}_{s}=20, \bar{k}_{s}=80$ and $R T T \approx 300 \mathrm{~ms}$.

[3] S. E. Salcudean, S. Tafazoli, P. D. Lawrence, and I. Chau, "Impedance Control of a Teleoperated Mini Excavator," IEEE Int. Conf. on Advanced Robotics, pp. 19-25, 1997.

[4] S. S. Haykin, Active Network Theory, AddisonWesley, 1970.

[5] F. B. Llewellyn, "Some Fundamental Properties of Transmission Systems," Proc. IRE,Vol. 40, pp. 271283, 1952.

[6] G. Raisbeck, "A Definition of Passive Linear Networks in Terms of Time and Energy," J. of Applied Physics, Vol. 25, 1954.

[7] B. Hannaford, "Stability and Performance Tradeoffs in Bi-Lateral Telemanipulation," IEEE Int. Conf. on Robotics and Automation, pp. 1764-1767, 1989.

[8] G. J. Raju, G. C. Verghese, and T. B. Sheridan, "Design Issues in 2-Port Network Models of Bilateral Remote Manipulation," IEEE Int. Conf. on Robotics and Automation, pp. 1316-1321, 1989.

[9] M. Shi, G. Tao, S. Graves, and J. H. Downs "Positive Realness and Tracking of Teleoperation Systems," IEEE Conf. on Decision and Control, pp. 2527-2532, 1998.

[10] R. J. Adams and B. Hannaford, "Stable Haptic Interaction with Virtual Environments," IEEE Trans. on Robotics and Automation, Vol. 15, No. 3, pp. 465474, 1999 .

[11] T. Fjallbrant, "Activity and Stability of Linear Networks," Trans. IEEE, CT-12, 1965.

[12] H. Kazerooni, "On the Robot Compliant Motion Control," ASME J. Dynamic Systems, Measurement, and Control, pp. 416-425, 1989.

[13] R. W. Daniel and P. R. McAree, "Fundamental Limits of Performance for Force Reflecting Teleoperation,” Int. J. Robotics Research, pp. 811-830, 1998.

[14] D. A. Lawrence, "Stability and Transparency in Bilateral Teleoperation," IEEE Trans. on Robotics and Automation, Vol. 9, No. 5, pp. 624-637, 1993. 